\title{
A retrospective examination of mean relative telomere length in the Tasmanian Familial Hematological Malignancies Study
}

\author{
NICHOLAS B. BLACKBURN ${ }^{1 *}$, JAC C. CHARLESWORTH $^{1,2^{*}}$, JAMES R. MARTHICK $^{1}$, \\ ELIZABETH M. TEGG ${ }^{3,4}$, KATHERINE A. MARSDEN ${ }^{1,3}$, VELANDAI SRIKANTH ${ }^{5}$, \\ JOHN BLANGERO ${ }^{2}$, RAY M. LOWENTHAL ${ }^{1,3}$, SIMON J. FOOTE ${ }^{6}$ and JOANNE L. DICKINSON ${ }^{1}$
}

\begin{abstract}
${ }^{1}$ Menzies Research Institute Tasmania, University of Tasmania, Hobart, TAS 7000, Australia; ${ }^{2}$ Department of Genetics, Texas Biomedical Research Institute, San Antonio, TX 78245-0549, USA; ${ }^{3}$ Royal Hobart Hospital, Hobart, TAS 7001;

${ }^{4}$ School of Medicine, University of Tasmania, Hobart, TAS 7000; ${ }^{5}$ Department of Medicine, Monash Medical Centre, Faculty of Medicine, Nursing and Health Sciences, Monash University, Clayton, VIC 3168;

${ }^{6}$ John Curtain School of Medical Research, Australian National University, ACT 2601, Australia
\end{abstract}

Received September 3, 2014; Accepted October 2, 2014

DOI: 10.3892/or.2014.3568

\begin{abstract}
Telomere length has a biological link to cancer, with excessive telomere shortening leading to genetic instability and resultant malignant transformation. Telomere length is heritable and genetic variants determining telomere length have been identified. Telomere biology has been implicated in the development of hematological malignancies (HMs), therefore, closer examination of telomere length in HMs may provide further insight into genetic etiology of disease development and support for telomere length as a prognostic factor in HMs. We retrospectively examined mean relative telomere length in the Tasmanian Familial Hematological Malignancies Study using a quantitative PCR method on genomic DNA from peripheral blood samples. Fifty-five familial HM cases, 191 unaffected relatives of familial HM cases and 75 non-familial HM cases were compared with 758 population controls. Variance components modeling was employed to identify factors influencing variation in telomere length. Overall, HM cases had shorter mean relative telomere length $\left(\mathrm{P}=2.9 \times 10^{-6}\right)$ and this was observed across both familial and non-familial HM cases $\left(\mathrm{P}=2.2 \times 10^{-4}\right.$ and $2.2 \times 10^{-5}$, respectively) as well as additional subgroupings of HM cases according to broad subtypes. Mean relative telomere length was also significantly heritable $\left(62.6 \% ; \mathrm{P}=4.7 \times 10^{-5}\right)$ in the $\mathrm{HM}$ families in the present study. We present new evidence of significantly shorter mean relative telomere length in both familial and non-familial HM
\end{abstract}

Correspondence to: Dr Joanne L. Dickinson, Menzies Research Institute Tasmania, University of Tasmania, Private Bag 23, 17 Liverpool Street, Hobart, TAS 7000, Australia

E-mail: jo.dickinson@utas.edu.au

${ }^{*}$ Contributed equally

Key words: telomere length, hematological malignancies, familial cancer cases from the same population adding further support to the potential use of telomere length as a prognostic factor in HMs. Whether telomere shortening is the cause of or the result of HMs is yet to be determined, but as telomere length was found to be highly heritable in our HM families this suggests that genetics driving the variation in telomere length is related to HM disease risk.

\section{Introduction}

Telomeres are DNA-protein structures at chromosome ends consisting of repeating hexameric nucleotide sequences of TTAGGG (1). The primary role of the telomere is to cap chromosome ends to prevent aberrant recombination as a result of exposed chromosomal DNA; making telomeres essential for maintenance of genomic integrity (2). With each cell division telomeres shorten due to the incomplete DNA replication of chromosome ends by DNA polymerases eventually triggering cell senescence or apoptosis to prevent further shortening and exposure of chromosomal DNA (3). The telomerase complex can counteract telomere shortening in actively dividing cells by catalyzing the addition of TTAGGG repeats to chromosome ends $(3,4)$, however, this is not a full restoration and telomeres progressively shorten with age $(3,5,6)$. A number of studies have reported an association between telomere length in lymphocytes and an increased risk of age-related diseases including cancer $(7,8)$. To date, the main understanding of the role of telomeres in cancer is that excessive telomere shortening leads to increased genetic instability and chromosomal end-to-end fusions $(2,9)$, which then leads to a malignant cell transformation (9).

Studies of monozygotic and dizygotic twins and large families have established a genetic component to the determination of telomere length. Estimates of the heritability of telomere length ranges between 78 and $82 \%$ in studies of twins and sibling pairs (which generally produce inflated heritability estimates) $(10,11)$ and $44 \%$ in a study of large Amish families (12). Although it has been proposed that the heritability of 
telomere length can be accounted for by shared environmental factors (13) the consensus is that telomere length is primarily determined by parental inheritance including at least partial inheritance of chromosome-specific telomere lengths $(14,15)$. This view is strongly supported by mouse models of telomere length inheritance (16).

Telomere length is a proposed risk factor for cancer given its importance in maintaining genomic integrity $(2,9)$ and shorter telomeres have been shown to be associated with a range of cancers $(7,8,17)$. The proposed role of telomere length as a cancer risk factor, coupled with its high heritability, raises the idea that telomere length may also be important in familial cancers such as familial hematological malignancies (HMs). Additionally, inherited changes in telomere length as a result of mutations in the telomere maintenance genes TERT and TERC have been identified in four HM families with myelodysplastic syndrome and acute myeloid leukemia (MDS-AML) (18). Therefore, telomere length may be a risk factor for other HM subtypes.

Hematological malignancies arise as a result of the neoplastic transformation of cells involved in hematopoiesis and include a broad range of subtypes of leukemias, lymphomas and myelomas (19). A well-established risk factor for HMs is family history indicating that familial HMs have an underlying genetic component. For example, a population-based study of the Swedish Cancer Registry showed an 8.5-fold increase in the risk of developing chronic lymphocytic leukemia (CLL) in first-degree relatives of CLL patients as well as an increased risk of developing other HM subtypes providing evidence for a shared genetic etiology across HM subtypes (20).

There is accumulating evidence that implicates telomere length as an important factor in the development of a range of HMs. This includes studies of several different HM subtypes where shorter telomeres were found in circulating tumor cells (21-26). While these studies have revealed important insights into telomere dynamics in circulating tumor cells there has been less focus on prospective and retrospective studies of pre-disease and remission telomere lengths respectively in HM patients. Indeed, one prospective study of telomere length in pre-disease blood samples surprisingly showed that longer telomere length was associated with a future risk of NHL (nonHodgkin lymphoma) (27). Furthermore, in a study of chronic leukemia, Mansouri and colleagues (28) elegantly showed that telomere length has potential as a clinical prognostic marker in HMs. In their study, patients with shorter telomeres were associated with high-risk genetic markers and in patients with otherwise good prognostic markers, telomere length was an independent prognostic factor that subdivided the good prognosis group into groups with distinct outcomes. Therefore, there is potential for telomere length to be a clinically relevant prognostic risk factor for HMs.

The aim of the present study was to explore whether telomere length is involved in familial HMs and to find new evidence supporting telomere length as a prognostic risk factor for HMs. To this end, we examined telomere length in the Tasmanian Familial Hematological Malignancies Study (TFHMS); a genetic study comprising large Tasmanian families with multiple cases of HMs and a collection of population matched non-familial HM cases (29-31) and controls $(32,33)$. Previously, similar collections have focused on families with one predominant subtype of HM. The TFHMS includes families with a dense aggregation of several HM subtypes across multiple generations; for example family LK2042 includes 32 cases in five generations (Table I).

The strength of a familial approach to examining telomere length lies in the enrichment of the shared genetic backgrounds between related individuals as related cases are likely to share genetic variants contributing to variation in telomere length which may in turn be affecting their risk of developing HMs. In the present study, we used the TFHMS to measure the heritability of telomere length as a quantitative trait in the study families and then examine whether HMs account for measured variation in telomere length.

\section{Materials and methods}

Ethics statement. The TFHM study was approved by the Human Research Ethics Committee (Tasmanian network), reference number: H8551, and written informed consent was obtained from all participating individuals.

The Tasmanian Familial Hematological Malignancies Study. As previously described (29) during the period 1972-1980 all patients with HMs diagnosed in Tasmania (the island state of Australia) were invited to participate in a population-based study examining the association of occupation and place of residence with risk of development of myeloproliferative and lymphoproliferative disorders $(34,35)$. Using a genealogical database at the Menzies Research Institute Tasmania the individuals participating in the original population-based study were linked to both current generations and records from the Tasmanian Cancer Registry, which has documented cases of HMs since 1978. Family members provided further information through questionnaires and personal interviews. This allowed us to form pedigrees of Tasmanian families with multiple cases of HMs as well as a collection of HM cases with no reported family history of disease (Table I).

Confirmation of diagnosis was, where possible, obtained for all cases and in particular 13 study families were classified by a single experienced hematologist (E.M.T.) according to the 2008 World Health Organization classification (19) as previously described (29). For the remaining study families, case diagnosis was obtained from the Tasmanian Cancer Registry records and by review of available pathology reports of cases that consented to participate in the TFHMS. More extensive clinical information is not currently available due to the multi-center and multi-specialist nature of the original data collection.

Study samples. In this TFHM-based study we used DNA obtained from peripheral blood samples from 55 familial HM cases, 191 unaffected relatives of familial cases and 75 nonfamilial cases. DNA from 40 TFHMS families was available for the present study with samples available from both HM cases and unaffected relatives in 14 families. The remaining families were comprised of samples from HM cases with a known family history of disease alone or from unaffected relatives of HM cases. Of the 191 unaffected relatives, 171 were first-degree relatives of HM cases and the remaining subjects were more distantly related or spouses. For HM cases, 
Table I. Summary of the TFHMS families used in this study.

\begin{tabular}{|c|c|c|c|c|}
\hline Family & $\begin{array}{l}\text { Known } \\
\text { HM } \\
\text { cases }\end{array}$ & $\begin{array}{c}\text { Generations } \\
\text { with } \\
\text { HM } \\
\text { cases }\end{array}$ & $\begin{array}{c}\text { HM cases } \\
\text { with } \\
\text { telomere } \\
\text { length } \\
\text { measurement }\end{array}$ & $\begin{array}{l}\text { Unaffected } \\
\text { relatives } \\
\text { with telomere } \\
\text { length } \\
\text { measurement }\end{array}$ \\
\hline LK0001 & 14 & 4 & 1 & 16 \\
\hline LK0002 & 15 & 3 & 1 & 5 \\
\hline LK0004 & 7 & 2 & 1 & 11 \\
\hline LK0016 & 18 & 5 & 2 & 19 \\
\hline LK0024 & 3 & 2 & 1 & 0 \\
\hline LK0026 & 6 & 2 & 1 & 5 \\
\hline LK0040 & 7 & 4 & 2 & 2 \\
\hline LK0051 & 21 & 5 & 3 & 26 \\
\hline LK0054 & 9 & 3 & 0 & 2 \\
\hline LK0065 & 8 & 2 & 0 & 8 \\
\hline LK0124 & 24 & 5 & 2 & 34 \\
\hline LK0132 & 5 & 2 & 0 & 7 \\
\hline LK0139 & 7 & 2 & 1 & 2 \\
\hline LK0153 & 9 & 2 & 3 & 2 \\
\hline LK0511 & 2 & 2 & 1 & 0 \\
\hline LK0512 & 2 & 1 & 1 & 0 \\
\hline LK0537 & 2 & 1 & 2 & 0 \\
\hline LK0546 & 2 & 2 & 1 & 0 \\
\hline LK0560 & 2 & 2 & 1 & 0 \\
\hline LK0561 & 2 & 2 & 1 & 0 \\
\hline LK0600 & 5 & 3 & 2 & 0 \\
\hline LK0625 & 4 & 2 & 2 & 0 \\
\hline LK0647 & 2 & 2 & 1 & 0 \\
\hline LK0672 & 3 & 3 & 1 & 0 \\
\hline LK0836 & 6 & 3 & 2 & 5 \\
\hline LK1155 & 2 & 1 & 1 & 3 \\
\hline LK2042 & 32 & 5 & 6 & 40 \\
\hline LK2447 & 3 & 2 & 1 & 2 \\
\hline LK6000 & 6 & 2 & 1 & 0 \\
\hline LK7739 & 2 & 1 & 1 & 0 \\
\hline LK7740 & 2 & 2 & 2 & 0 \\
\hline LK7743 & 3 & 2 & 2 & 0 \\
\hline LK7744 & 2 & 2 & 0 & 1 \\
\hline LK7748 & 2 & 2 & 1 & 0 \\
\hline LK7749 & 3 & 2 & 1 & 0 \\
\hline LK7750 & 4 & 2 & 2 & 0 \\
\hline LK7751 & 9 & 3 & 1 & 0 \\
\hline LK7754 & 3 & 1 & 1 & 0 \\
\hline LK7755 & 2 & 2 & 1 & 0 \\
\hline LK7768 & 2 & 1 & 1 & 0 \\
\hline $\begin{array}{l}\text { Non-familial } \\
\text { cases }\end{array}$ & al - & - & 75 & 1 \\
\hline
\end{tabular}

HM, hematological malignancy; TFHM, Tasmanian Familial Hematological Malignancies Study.

DNA was collected from 1 month to 64.9 years post HM diagnosis (mean, 9.9 years). Population controls were recruited randomly from the Tasmanian electoral role $(n=758)$ through the TASCOG study (33) (a population-based study of gait in older Tasmanians) or obtained from the control samples in a Tasmanian familial prostate cancer case-control study (32) both conducted at the Menzies Research Institute Tasmania. Details concerning the participants in this study are shown in Table II, and the distribution of HM case subtypes in this study is summarized in Table III. Non-familial HM cases had no self-reported family history of HMs and did not appear in any of our study families after thorough genealogical examination. Frequent updates from the Tasmanian Cancer Registry were used to monitor the occurrence of HMs in the unaffected relatives that are part of this study. Population control DNA samples were also extracted from peripheral blood samples in the same laboratory, using the same methodology as the TFHMS samples. Genomic DNA was extracted from peripheral blood samples using the Nucleon BACC 3 DNA Extraction kit (GE Healthcare).

Telomere length measurement. We investigated the mean relative telomere length in peripheral blood samples using a slightly amended protocol for a validated monochrome multiplex quantitative PCR method outlined by Cawthon (36). This method measures the relative telomere length by calculating the ratio, T/S, between telomere repeat copy number amplification (T) and the amplification of a single-copy gene, albumin (S). The average $\mathrm{T} / \mathrm{S}$ ratio was obtained as the mean of the triplicate measurements for each sample. Individual measurements were excluded from the average $\mathrm{T} / \mathrm{S}$ ratio calculation when the replicate failed or a large standard error was observed. The coefficient of variation calculated across all assay plates using repeated cross-plate samples was $3.4 \%$.

Telomere length measurement was performed in $10 \mu \mathrm{l}$ volumes using a LightCycler 480 in a 96 -well plate format. Each 96-well plate contained a six point standard curve 2, 5, $15,50,100$ and $150 \mathrm{ng}$, a unique sample common to each plate, a no template control and 24 unknown case/control samples all repeated in triplicate, with $1.6 \%$ sample replication across plates. The genomic DNA used for the standard curve was from a 27-year-old female control study participant.

Final reagent concentrations were $5 \mathrm{ng}$ of genomic DNA, primer telg $200 \mathrm{nM}$ (5'-ACACTAAGGTTTGGGTTTGGGT TTGGGTTTGGGTTAGTGT-3'), primer telc 700 nM (5'-TG TTAGGTATCCCTATCCCTATCCCTATCCCTATCCCTAA CA-3'), primer albu $500 \mathrm{nM}$ (5'-CGGCGGCGGGCGGCG CGGGCTGGGCGGAAATGCTGCACAGAATCCTTG-3'), primer albd $500 \mathrm{nM}$ (5'-GCCCGGCCCGCCGCGCCCG TCCCGCCGGAAAAGCATGGTCGCCTGTT-3'), AmpliTaq Gold (Applied Biosystems) 0.625 U, GeneAmp 10X PCR buffer (Life Technologies) containing $50 \mathrm{mM} \mathrm{KCl}, 10 \mathrm{mM}$ Tris- $\mathrm{HCl} \mathrm{pH} 8.3$ and $1.5 \mathrm{mM} \mathrm{MgCl}_{2}, 1 \mathrm{mM}$ DTT, $1 \mathrm{M}$ Betaine (Sigma-Aldrich), $0.0025 \mathrm{mM}$ Syto9 (Life Technologies) and $0.25 \mathrm{mM}$ of each dNTP (Bioline). Cycling conditions were as follows: $95^{\circ} \mathrm{C}$ for $15 \mathrm{~min}, 2$ cycles of $94^{\circ} \mathrm{C}$ for $15 \mathrm{sec}, 49^{\circ} \mathrm{C}$ for $60 \mathrm{sec}$; four cycles of $84^{\circ} \mathrm{C}$ for $20 \mathrm{sec}, 59^{\circ} \mathrm{C}$ for $30 \mathrm{sec}$, then 40 cycles of $94^{\circ} \mathrm{C}$ for $15 \mathrm{sec}, 59^{\circ} \mathrm{C}$ for $30 \mathrm{sec}$ with signal acquisition for telomere repeat copy number amplification, $84^{\circ} \mathrm{C}$ for $30 \mathrm{sec}$, then $85^{\circ} \mathrm{C}$ for $20 \mathrm{sec}$ with signal acquisition for albumin amplification. A melting curve was generated for each plate. $\mathrm{Ct}$ values were calculated using LinRegPCR (37) and a standard 
Table II. Mean age, sex distribution and relative telomere length in the sample groups.

\begin{tabular}{lcccc}
\hline Sample group & $\mathrm{N}$ & Male sex, $\mathrm{n}(\%)$ & Mean age (range) & Mean relative T/S ratio ${ }^{\mathrm{a}}(95 \% \mathrm{CI})$ \\
\hline Controls & 758 & $578(76.3)$ & $67.51(30.67-87.97)$ & $0.64(0.62-0.66)$ \\
Unaffected relatives of HM cases & 191 & $77(40.3)$ & $61.65(27.26-92.95)$ & $0.73(0.69-0.76)$ \\
All HM cases & 130 & $73(56.2)$ & $65.14(13.24-95.53)$ & $0.53(0.50-0.56)$ \\
Familial HM cases & 55 & $32(58.2)$ & $64.45(13.24-87.45)$ & $0.57(0.52-0.63)$ \\
Non-familial HM cases & 75 & $41(54.7)$ & $68.79(22.42-95.53)$ & $0.50(0.46-0.53)$ \\
\hline
\end{tabular}

${ }^{a}$ Mean relative T/S ratio is the ratio between telomere repeat copy number (T) and a single-copy gene, $A L B$, copy number (S), a measure of mean relative telomere length. CI, confidence interval; HM, hematological malignancy. Mean age (range) is expressed in years.

Table III. Disease characteristics of study samples.

HM familial cases, n (\%) HM non-familial cases, n (\%) All HM cases, n (\%)

\begin{tabular}{|c|c|c|c|}
\hline \multicolumn{4}{|l|}{ HM subtypes } \\
\hline Acute lymphoblastic leukemia & $2(3.6)$ & 0 & $2(1.5)$ \\
\hline Acute myeloid leukemia & $5(9.1)$ & $8(10.7)$ & $13(10.0)$ \\
\hline Chronic myeloid leukemia & 0 & $3(4.0)$ & $3(2.3)$ \\
\hline Essential thrombocythemia & $1(1.8)$ & $1(1.3)$ & $2(1.5)$ \\
\hline Hodgkin lymphoma & $5(9.1)$ & $4(5.3)$ & $9 \quad(6.9)$ \\
\hline Myelodysplastic syndrome & $2(3.6)$ & 0 & $2(1.5)$ \\
\hline Myeloproliferative neoplasm & 1 (1.8) & $2(2.7)$ & $3(2.3)$ \\
\hline T-cell non-Hodgkin lymphoma & 1 (1.8) & $2(2.7)$ & $3(2.3)$ \\
\hline \multicolumn{4}{|l|}{ Mature B cell neoplasms } \\
\hline Non-Hodgkin lymphoma unclassified & $2(3.6)$ & $10(13.3)$ & $12(9.2)$ \\
\hline Chronic lymphocytic leukemia & $12(21.8)$ & $12(16.0)$ & $24(18.5)$ \\
\hline Diffuse large B-cell lymphoma & $4(7.3)$ & $10(13.3)$ & $14(10.8)$ \\
\hline Follicular lymphoma & $4(7.3)$ & $9(12.0)$ & $13(10.0)$ \\
\hline Multiple myeloma & $7(12.7)$ & $5 \quad(6.7)$ & $12(9.2)$ \\
\hline Other $^{\mathrm{a}}$ & $9(16.4)$ & $9(12.0)$ & $18(13.8)$ \\
\hline Total & 55 & 75 & 130 \\
\hline
\end{tabular}

${ }^{a}$ Other includes Burkitt lymphoma, hairy cell leukemia, lymphoma of mucosa-associated lymphoid tissue and Waldenström macroglobulinemia. HM, hematological malignancy.

curve was generated for both the telomere and albumin PCRs. A linear regression of the standard curve measurement values was used to correct for any variation in fluorescence levels derived from small fluctuations in DNA concentration. The equations from the linear regression of each standard curve were then used to calculate the $\log$ (DNA) value for the unknown case/control samples.

Statistical analysis. Average T/S ratios greater than 4 standard deviations from the control mean were excluded as outliers. Mean relative T/S ratios with $95 \%$ confidence intervals (CI) are reported in Table II. For analysis mean relative T/S ratios were transformed to fit a normal distribution using the inversenormalization option in SOLAR (version 6.6.2) $(38,39)$ to prevent non-normal distribution errors. In order to fully utilize the extended pedigree study design, correct for relatedness, and to maximize the information provided by telomere length as a quantitative trait we used variance components modeling in $\operatorname{SOLAR}(38,39)$ to determine the heritability of telomere length (adjusting for kinship and significant covariates) and to calculate the association between telomere length and disease. The primary benefit to using SOLAR is its ability to incorporate relatedness through the use of a kinship matrix and to fully utilize the quantitative trait data, which increases the power and accuracy of the trait heritability calculation.

Sex, age, age $^{2}$ and their interactions were included as covariates in all relevant analyses. Potential batch effects were adjusted for by applying household modeling $(38,39)$ by coding each assay plate as a separate household. SOLAR has been previously used in the analysis of telomere length in related 


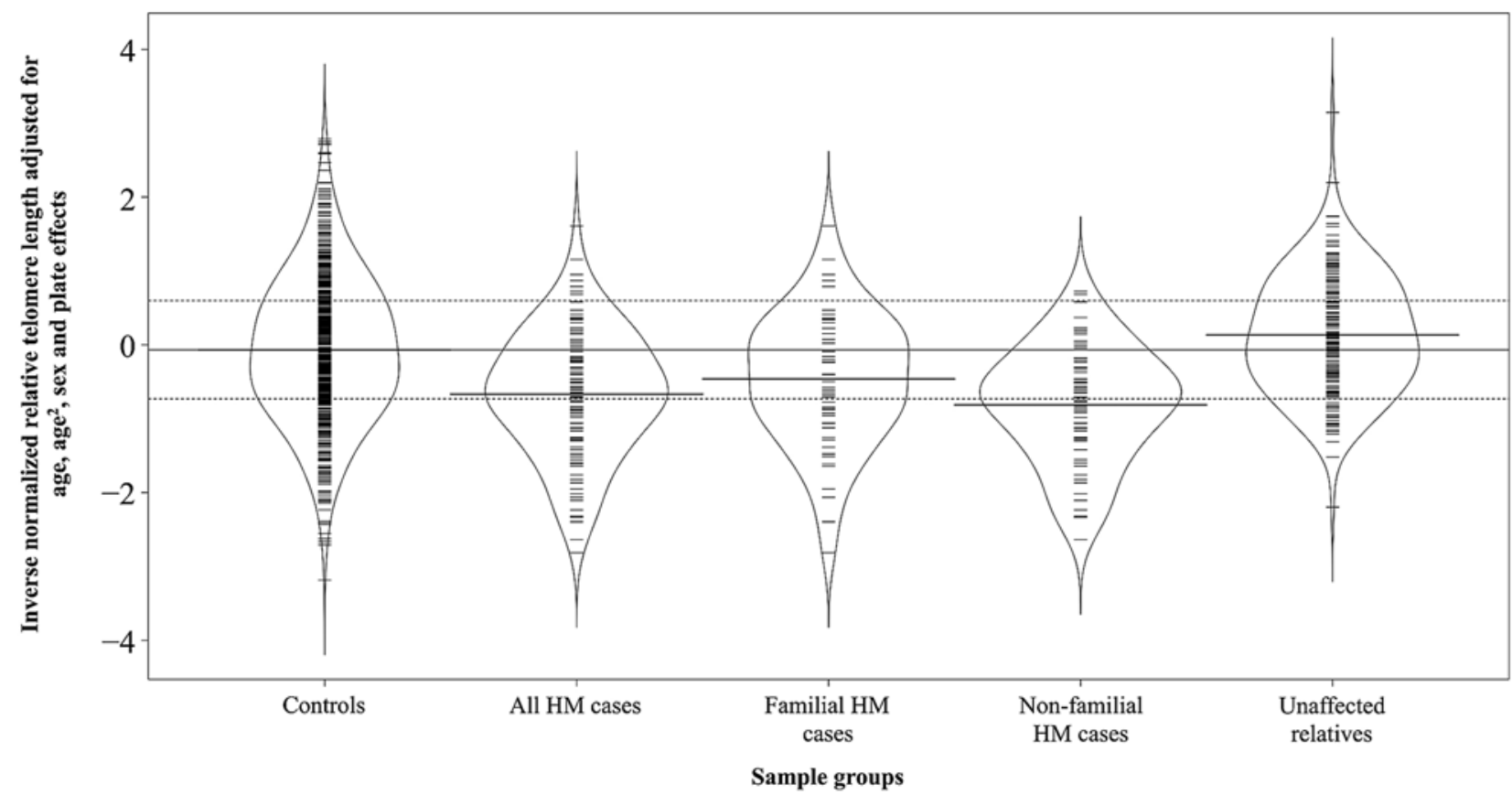

Figure 1. Bean plot quartile analysis of adjusted inverse normalized relative telomere lengths. The adjusted inverse normalized relative telomere length for each group is displayed as a bean plot with individual sample measurements as lines within the bean and the overall distribution of all samples in each group shown. Horizontal bars for each bean indicate the mean of each group. The solid line and dashed lines show the mean and interquartile range of the control group.

individuals $(11,12,40)$. The algorithms utilized in SOLAR for the analysis of quantitative traits in related individuals are more appropriate to employ in the present study than a more traditional approach of analyzing quantitative traits using percentiles or quartiles. Nevertheless, we also present observations from a quartile analysis of inverse normalized relative T/S ratios adjusted for age, sex and batch effects in SOLAR with quartiles defined from the adjusted T/S ratios in the control population (Fig. 1). Bean plots in Fig. 1 were constructed using the R package 'beanplot' (41).

\section{Results}

Mean relative telomere length in familial and non-familial HM cases, unaffected relatives and control subjects was measured by monochrome multiplex quantitative PCR. Using SOLAR we found that the heritability of mean relative telomere length was $62.5 \%\left(\mathrm{P}=4.7 \times 10^{-5}, \mathrm{SE}=0.14\right)$. The removal of $\mathrm{HM}$ cases $(n=130)$ from analysis only marginally altered the heritability of mean relative telomere length $\left(75.5 \% ; \mathrm{P}=1.2 \times 10^{-5}, \mathrm{SE}=0.15\right)$.

The use of variance components modeling in SOLAR permits appropriate statistical analyses inclusive of familial relationships. These analyses revealed that disease status was significantly associated with mean relative telomere length (Table IV, primary analysis model $1 ; \mathrm{P}=2.9 \times 10^{-6}$ ) with HM cases having shorter mean relative telomere length when compared with unaffected individuals. We conducted a separate analysis distinguishing familial and non-familial cases. Familial cases and non-familial cases each had significantly shorter mean relative telomere length (Table IV; primary analysis model $2, \mathrm{P}=2.2 \times 10^{-4}$ and $\mathrm{P}=2.2 \times 10-5$, respectively).

The most frequent type of HM diagnosed in the present study was mature B cell neoplasms (MBCNs; Table III).
Analysis of MBCNs as one group and HMs other than MBCNs as a second group (combined due to the small numbers of other subtypes) showed that both groupings had shorter mean relative telomere length than unaffected individuals from both study families and population controls (Table IV; primary analysis model $3, \mathrm{P}=3.5 \times 10^{-5}$ and $\mathrm{P}=9.3 \times 10^{-5}$, respectively). These groupings were then divided according to whether the HM case was familial or non-familial. Analysis showed that all HM case subgroupings maintained significantly shorter mean relative telomere length (Table IV; primary analysis model 4). An analysis using specific HM subtypes was not possible due to not having enough statistical power at this level of HM classification with small numbers of HM subtypes (Table III).

Variance components modeling also identified age and sex (Table IV; primary analysis model $1, \mathrm{P}=4.8 \times 10^{-8}$ and $\mathrm{P}=4.0 \times 10^{-3}$, respectively) as significant covariates for mean relative telomere length variation across all models. Mean relative telomere length declined with age and males had shorter telomeres than females. Age $^{2}$ was also a significant covariate, indicating that the decline in mean relative telomere length with age has a non-linear component (Table III; primary analysis model $1, \mathrm{P}=8.0 \times 10^{-3}$ ).

Four sub-analyses of the primary data were also performed to determine whether particular features of the study population were contributing to the disease associations found in the primary analysis models (Table IV). Sub-analyses included exclusion of HM cases, controls and unaffected relatives 80 years or older $(n=126)$, exclusion of CLL cases $(n=24)$, exclusion of cases with samples collected within two years of diagnosis $(n=24)$ as well as all three exclusions together $(n=162$, some individuals were in multiple exclusion categories). In each sub-analysis the principle findings from the primary analysis models were maintained. 
Table IV. Variance component modeling analysis of inverse normalized mean relative telomere length primary analysis and sub-analyses with exclusions.

\begin{tabular}{|c|c|c|c|c|c|}
\hline Models and variables & $\begin{array}{l}\text { Primary } \\
\text { analysis } \\
\text { P-values }\end{array}$ & $\begin{array}{c}\geq 80 \text { years old } \\
\text { excluded } \\
(n=126) \\
\text { P-values }\end{array}$ & $\begin{array}{l}\text { CLL cases } \\
\text { excluded } \\
(\mathrm{n}=24) \\
\text { P-values }\end{array}$ & $\begin{array}{c}\text { Possible } \\
\text { malignant samples } \\
\text { excluded }^{\mathrm{a}} \\
(\mathrm{n}=24) \\
\text { P-values }\end{array}$ & $\begin{array}{c}\text { All exclusions } \\
\text { applied } \\
(\mathrm{n}=162) \\
\text { P-values }\end{array}$ \\
\hline \multicolumn{6}{|l|}{ Model 1} \\
\hline Age & $4.8 \times 10^{-8}$ & $7.5 \times 10^{-5}$ & $1.6 \times 10^{-8}$ & $6.9 \times 10^{-8}$ & $3.4 \times 10^{-5}$ \\
\hline $\mathrm{Age}^{2}$ & $8.0 \times 10^{-3}$ & 0.04 & $6.0 \times 10^{-3}$ & 0.01 & 0.07 \\
\hline Sex & $4.0 \times 10^{-3}$ & 0.01 & $7.0 \times 10^{-3}$ & $2.0 \times 10^{-3}$ & 0.01 \\
\hline All HM cases & $2.9 \times 10^{-6}$ & $7.3 \times 10^{-6}$ & $2.9 \times 10^{-7}$ & $1.1 \times 10^{-4}$ & $4.6 \times 10^{-5}$ \\
\hline$\%$ trait variance accounted for by model & $10.07 \%$ & $9.46 \%$ & $10.38 \%$ & $9.56 \%$ & $9.38 \%$ \\
\hline \multicolumn{6}{|l|}{ Model 2} \\
\hline Age & $4.3 \times 10^{-8}$ & $5.1 \times 10^{-5}$ & $1.6 \times 10^{-8}$ & $7.4 \times 10^{-8}$ & $3.7 \times 10^{-5}$ \\
\hline $\mathrm{Age}^{2}$ & $8.0 \times 10^{-3}$ & 0.04 & $6.0 \times 10^{-3}$ & 0.01 & 0.07 \\
\hline Sex & $3.0 \times 10^{-3}$ & 0.01 & $6.0 \times 10^{-3}$ & $2.0 \times 10^{-3}$ & $9.0 \times 10^{-3}$ \\
\hline Familial HM cases & $2.2 \times 10^{-4}$ & $1.0 \times 10^{-3}$ & $1.6 \times 10^{-5}$ & 0.01 & $8.0 \times 10^{-3}$ \\
\hline Non-familial HM cases & $2.2 \times 10^{-5}$ & $6.9 \times 10^{-6}$ & $7.1 \times 10^{-5}$ & $3.3 \times 10^{-5}$ & $2.7 \times 10^{-5}$ \\
\hline$\%$ trait variance accounted for by model & $10.62 \%$ & $10.29 \%$ & $10.55 \%$ & $10.48 \%$ & $10.00 \%$ \\
\hline \multicolumn{6}{|l|}{ Model 3} \\
\hline Age & $4.7 \times 10^{-8}$ & $7.2 \times 10^{-5}$ & $1.7 \times 10^{-8}$ & $7.7 \times 10^{-8}$ & $3.7 \times 10^{-5}$ \\
\hline $\mathrm{Age}^{2}$ & $8.0 \times 10^{-3}$ & 0.04 & $6.0 \times 10^{-3}$ & 0.01 & 0.07 \\
\hline Sex & $4.0 \times 10^{-3}$ & 0.01 & $6.0 \times 10^{-3}$ & $2.0 \times 10^{-3}$ & 0.01 \\
\hline MBCNs & $3.5 \times 10^{-5}$ & $7.8 \times 10^{-5}$ & $5.5 \times 10^{-6}$ & $5.7 \times 10^{-4}$ & $3.8 \times 10^{-4}$ \\
\hline HMs other than MBCNs & $9.3 \times 10^{-5}$ & $1.5 \times 10^{-4}$ & $3.4 \times 10^{-5}$ & $1.0 \times 10^{-3}$ & $5.8 \times 10^{-4}$ \\
\hline$\%$ trait variance accounted for by model & $10.08 \%$ & $9.50 \%$ & $10.37 \%$ & $9.56 \%$ & $9.39 \%$ \\
\hline \multicolumn{6}{|l|}{ Model 4} \\
\hline Age & $4.8 \times 10^{-8}$ & $4.1 \times 10^{-5}$ & $1.9 \times 10^{-8}$ & $6.6 \times 10^{-8}$ & $3.8 \times 10^{-5}$ \\
\hline $\mathrm{Age}^{2}$ & $9.0 \times 10^{-3}$ & 0.05 & $7.0 \times 10^{-3}$ & 0.02 & 0.08 \\
\hline Sex & $3.0 \times 10^{-3}$ & 0.01 & $6.0 \times 10^{-3}$ & $2.0 \times 10^{-3}$ & $9.0 \times 10^{-3}$ \\
\hline Familial MBCNs & 0.02 & 0.04 & $3.0 \times 10^{-3}$ & 0.18 & 0.07 \\
\hline Familial cases other than MBCNs & $5.2 \times 10^{-4}$ & $3.0 \times 10^{-3}$ & $5.7 \times 10^{-4}$ & 0.01 & 0.04 \\
\hline Non-familial MBCNs & $2.4 \times 10^{-5}$ & $1.5 \times 10^{-5}$ & $1.3 \times 10^{-4}$ & $4.8 \times 10^{-5}$ & $1.5 \times 10^{-4}$ \\
\hline Non-familial cases other than MBCNs & $2.0 \times 10^{-3}$ & $4.2 \times 10^{-4}$ & $3.0 \times 10^{-3}$ & $2.0 \times 10^{-3}$ & $3.2 \times 10^{-4}$ \\
\hline$\%$ trait variance accounted for by model & $10.87 \%$ & $10.58 \%$ & $10.57 \%$ & $10.45 \%$ & $10.05 \%$ \\
\hline
\end{tabular}

P-values for the significance of each trait or covariate were derived from variance component polygenic modeling in SOLAR. ${ }^{a} \mathrm{HM}$ case samples collected within \pm 2 years of diagnosis were excluded. CLL, chronic lymphocytic leukemia; HM, hematological malignancy; MBCNs, mature B-cell neoplasms.

Categorization of cases into quartiles of mean relative telomere length determined from the distribution of age, sex and batch effect adjusted mean relative telomere length in controls (Fig. 1) showed that $43.1 \%$ of HM cases were in the lowest quartile of mean relative telomere length (below the lower interquartile dashed line), with $36.4 \%$ of familial HM cases and $48 \%$ of non-familial HM cases in the lowest quartile, whilst only $13.1 \%$ of unaffected relatives were in the lowest quartile. Similarly a low percentage of cases $(5.4 \%)$ were in the longest quartile of mean relative telomere length (above the upper interquartile dashed line) whereas $28.3 \%$ of unaffected relatives were in the longest quartile. A clear trend for shorter mean relative telomere length in a higher percentage of HM cases was observed but this analysis did not permit familial relationships to be included in the analysis.

\section{Discussion}

These analyses determined that mean relative telomere length is highly heritable within the TFHMS families supporting previously reported heritability estimates in non-disease families (10-12). Our finding that mean relative telomere length was shorter in both familial and non-familial HM cases indicates that telomere length is likely to be important 
in the genetic etiology of HMs. A previous study of mean relative telomere length in familial myelodysplastic syndrome MDS-AML has shown that affected individuals from four small families had shorter telomeres concurrent with mutations in the telomerase gene TERT and its RNA component TERC (18). Of the five cases across the four families reported to have shorter telomeres, two had aplastic anemia, two had MDS and one had MDS-AML. The present study extends the findings of Kirwan and colleagues (18) to that of large families with multiple HM subtypes finding new evidence of the involvement of telomere length in both familial and nonfamilial HMs.

Age, sex and age $^{2}$ as covariates explained a proportion of the variation in mean relative telomere length in the present study. This is in keeping with telomere length declining with age and males having shorter telomeres than females (7). A significant age ${ }^{2}$ indicates a non-linear component in the age-related telomere length decline, a finding in line with a recent report showing a differential rate of decrease in telomere length over different age ranges (42). Our population controls did have a higher percentage of males, which could be suggested to be driving the association with sex, however, SOLAR was used to correct the mean relative telomere length for sex effects.

An important caveat with our retrospective study is that the finding of shorter mean relative telomere lengths in HM cases could also be related to disease susceptibility, treatment or the disease process. The present study did not have the necessary clinical information to appropriately analyze these factors. Currently, the literature surrounding the role of chemotherapeutic agents in telomere shortening remains controversial and inconclusive. Several studies in both HMs and other cancers such as breast cancer have shown that telomere length is unaffected when comparing pre- and post-chemotherapy measurements, when comparing patients who receive chemotherapy to those that do not or when comparing telomere length between patients and population controls $(25,28,43,44)$. Other studies show a heterogeneous effect of chemotherapy on telomere length (45-47). It could be concluded from these reports and others that chemotherapy has no consistent influence on telomere length in blood cells particularly when examining multiple chemotherapeutic treatment regimens.

A second consideration is that shorter telomeres in HM cases could be the result of malignant cell DNA within the genomic DNA sample. We recognize that circulating malignant cells can be present for many years in chronic HM subtypes such as CLL. Based on the clinical diagnoses of HM cases in our study, we conducted two additional sub-analyses of the primary data. In the one analysis we removed all CLL cases $(n=24)$ on the basis that DNA obtained from blood of cases with this subtype of HM was likely to contain DNA from diseased cells (Table IV). In the second analysis we removed all cases for which blood samples were obtained for DNA within 2 years of diagnosis ( $n=24$; Table IV). Repeating the variance components modeling in these two analyses maintained the key significant associations with HM disease, suggesting that circulating disease did not contribute to the telomere length associations we have identified. In an additional sub-analysis we excluded all HM cases, controls and unaffected relatives $(n=126)$ aged 80 years and above on the basis that the population HM risk increases with age. This did not change the principle findings of shorter telomeres in familial and non-familial HM cases nor did a final combined sub-analysis excluding individuals from all 3 sub-analyses. All cases, controls and unaffected relatives were included in the primary analysis models reported in Table IV.

In conclusion, our analyses showed for the first time that mean relative telomere length is heritable in large HM families with multiple generations affected by multiple subtypes of HMs, indicating a strong genetic effect driving trait variation. We also showed that both familial and non-familial HM cases from the same population had shorter mean relative telomere length. Taken together, the results from this retrospective study provide new evidence that mean relative telomere length is an important genetic factor in a wide range of HM subtypes and in individuals with and without a family history of disease. These findings contribute further support to the use of telomere length as a prognostic risk factor for HMs

\section{Acknowledgements}

The authors thank Annette Banks for her ongoing genealogical support. The authors would also like to thank the many years of dedicated research performed by Anne Piaszczyk, and the late Jean Panton. Finally we acknowledge the participants and their families in the Tasmanian Familial Hematological Malignancies study.

\section{References}

1. Moyzis RK, Buckingham JM, Cram LS, et al: A highly conserved repetitive DNA sequence, (TTAGGG)n, present at the telomeres of human chromosomes. Proc Natl Acad Sci USA 85: 6622-6626, 1988.

2. Counter CM, Avilion AA, LeFeuvre CE, Stewart NG, Greider CW, Harley CB and Bacchetti S: Telomere shortening associated with chromosome instability is arrested in immortal cells which express telomerase activity. EMBO J 11: 1921-1929, 1992.

3. Harley CB, Futcher AB and Greider CW: Telomeres shorten during ageing of human fibroblasts. Nature 345: 458-460, 1990.

4. Greider CW and Blackburn EH: Identification of a specific telomere terminal transferase activity in Tetrahymena extracts. Cell 43: 405-413, 1985.

5. Allsopp RC, Vaziri H,Patterson C, et al: Telomere length predicts replicative capacity of human fibroblasts. Proc Natl Acad Sci USA 89: 10114-10118, 1992.

6. Brouilette SW, Moore JS, McMahon AD, et al: Telomere length risk of coronary heart disease, and statin treatment in the West of Scotland Primary Prevention Study: a nested case-control study. Lancet 369: 107-114, 2007.

7. McGrath M, Wong JYY, Michaud D, Hunter DJ and de Vivo I: Telomere length, cigarette smoking, and bladder cancer risk in men and women. Cancer Epidemiol Biomarkers Prev 16: 815-819, 2007.

8. Willeit P, Willeit J, Mayr A, et al: Telomere length and risk of incident cancer and cancer mortality. JAMA 304: 69-75, 2010.

9. Artandi SE, Chang S, Lee SL, Alson S, Gottlieb GJ, Chin L and DePinho RA: Telomere dysfunction promotes non-reciprocal translocations and epithelial cancers in mice. Nature 406: 641-645, 2000.

10. Slagboom PE, Droog S and Boomsma DI: Genetic determination of telomere size in humans: a twin study of three age groups. Am J Hum Genet 55: 876-882, 1994.

11. Vasa-Nicotera M, Brouilette S, Mangino M, et al: Mapping of a major locus that determines telomere length in humans. Am J Hum Genet 76: 147-151, 2005.

12. Njajou OT, Cawthon RM, Damcott CM, et al: Telomere length is paternally inherited and is associated with parental lifespan. Proc Natl Acad Sci USA 104: 12135-12139, 2007. 
13. Huda N, Tanaka H, Herbert BS, Reed T and Gilley D: Shared environmental factors associated with telomere length maintenance in elderly male twins. Aging Cell 6: 709-713, 2007.

14. Graakjaer J, Bischoff C, Korsholm L, et al: The pattern of chromosome-specific variations in telomere length in humans is determined by inherited, telomere-near factors and is maintained throughout life. Mech Ageing Dev 124: 629-640, 2003.

15. Graakjaer J, Londoño-Vallejo JA, Christensen K and Kølvraa S: The pattern of chromosome-specific variations in telomere length in humans shows signs of heritability and is maintained through life. Ann NY Acad Sci 1067: 311-316, 2006.

16. Chiang YJ, Calado RT, Hathcock KS, Lansdorp PM, Young NS and Hodes RJ: Telomere length is inherited with resetting of the telomere set-point. Proc Natl Acad Sci USA 107: 10148-10153, 2010.

17. Wu X, Amos CI, Zhu Y, et al: Telomere dysfunction: a potential cancer predisposition factor. J Natl Cancer Inst 95: 1211-1218, 2003.

18. Kirwan MJ, Vulliamy T, Marrone A, et al: Defining the pathogenic role of telomerase mutations in myelodysplastic syndrome and acute myeloid leukemia. Hum Mutat 30: 1567-1573, 2009.

19. Swerdlow SH, Campo E, Harris NL, et al: WHO Classification of Tumours of Haematopoietic and Lymphoid Tissues. IARC, Lyon, 2008.

20. Goldin LR, Björkholm M, Kristinsson SY, Turesson I and Landgren O: Elevated risk of chronic lymphocytic leukemia and other indolent non-Hodgkin's lymphomas among relatives of patients with chronic lymphocytic leukemia. Haematologica 94: 647-653, 2009

21. Wu K, Lund M, Bang K and Thestrup-Pedersen K: Telomerase activity and telomere length in lymphocytes from patients with cutaneous T-cell lymphoma. Cancer 86: 1056-1063, 1999.

22. Terasaki Y, Okumura H, Ohtake S and Nakao S: Accelerated telomere length shortening in granulocytes: a diagnostic marker for myeloproliferative diseases. Exp Hematol 30: 1399-1404, 2002.

23. Wu KD, Orme LM, Shaughnessy J, Jacobson J, Barlogie B and Moore MAS: Telomerase and telomere length in multiple myeloma: correlations with disease heterogeneity, cytogenetic status, and overall survival. Blood 101: 4982-4989, 2003.

24. Hartmann U, Brümmendorf TH, Balabanov S, Thiede C, Illme T and Schaich M: Telomere length and hTERT expression in patients with acute myeloid leukemia correlates with chromosomal abnormalities. Haematologica 90: 307-316, 2005.

25. Ghaffari SH, Shayan-Asl N, Jamialahmadi AH, Alimoghaddam K and Ghavamzadeh A: Telomerase activity and telomere length in patients with acute promyelocytic leukemia: indicative of proliferative activity, disease progression, and overall survival. Ann Oncol 19: 1927-1934, 2008.

26. Capraro V, Zane L, Poncet D, et al: Telomere deregulations possess cytogenetic, phenotype, and prognostic specificities in acute leukemias. Exp Hematol 39: 195-202.e2, 2011.

27. Lan Q, Cawthon R, Shen M, et al: A prospective study of telomere length measured by monochrome multiplex quantitative PCR and risk of non-Hodgkin lymphoma. Clin Cancer Res 15: 7429-7433, 2009.

28. Mansouri L, Grabowski P, Degerman S, et al: Short telomere length is associated with NOTCH1/SF3B1/TP53 aberrations and poor outcome in newly diagnosed chronic lymphocytic leukemia patients. Am J Hematol 88: 647-651, 2013.

29. Tegg EM, Thomson RJ, Stankovich J, et al: Evidence for a common genetic aetiology in high-risk families with multiple haematological malignancy subtypes. Br J Haematol 150: $456-462,2010$
30. Tegg EM, Thomson RJ, Stankovich JM, et al: Anticipation in familial hematologic malignancies. Blood 117: 1308-1310, 2011.

31. Lowenthal RM, Tegg EM and Dickinson JL: The Familial Tasmanian Haematological Malignancies Study (FaTHMS): its origins, its history and the phenomenon of anticipation. Transfus Apher Sci 49: 113-115, 2013.

32. FitzGerald LM, Patterson B, Thomson R, et al: Identification of a prostate cancer susceptibility gene on chromosome $5 \mathrm{p} 13 \mathrm{q} 12$ associated with risk of both familial and sporadic disease. Eur J Hum Genet 17: 368-377, 2009.

33. Callisaya ML, Blizzard L, Schmidt MD, Martin KL, McGinley JL, Sanders LM and Srikanth VK: Gait, gait variability and the risk of multiple incident falls in older people: a population-based study. Age Ageing 40: 481-487, 2011.

34. Giles GG, Lickiss JN, Baikie MJ, Lowenthal RM and Panton J: Myeloproliferative and lymphoproliferative disorders in Tasmania, 1972-80: occupational and familial aspects. J Natl Cancer Inst 72: 1233-1240, 1984.

35. Lickiss JN, Giles GG, Baikie MJ, Lowenthal RM, Challis D and Panton J: Myeloproliferative and lymphoproliferative disorders in Tasmania, 1972-80: patterns in space and time. J Natl Cancer Inst 72: 1223-1231, 1984.

36. Cawthon RM: Telomere length measurement by a novel monochrome multiplex quantitative PCR method. Nucleic Acids Res 37: e21, 2009

37. Ruijter JM, Ramakers C, Hoogaars WMH, Karlen Y, Bakker O, van den Hoff MJB and Moorman AFM: Amplification efficiency: linking baseline and bias in the analysis of quantitative PCR data. Nucleic Acids Res 37: e45, 2009.

38. Almasy L and Blangero J: Multipoint quantitative-trait linkage analysis in general pedigrees. Am J Hum Genet 62: 1198-1211, 1998.

39. Almasy L and Blangero J: Variance component methods for analysis of complex phenotypes. Cold Spring Harb Protoc 2010: pdb.top77-pdb.top77, 2010.

40. Njajou OT, Blackburn EH, Pawlikowska L, et al: A common variant in the telomerase RNA component is associated with short telomere length. PLoS One 5: e13048, 2010.

41. Kampstra P: Beanplot: A boxplot alternative for visual comparison of distributions. J Statistical Software 28: 1-9, 2008. http:// www.jstatsoft.org/v28/c01/.

42. Aubert G, Baerlocher GM, Vulto I, Poon SS and Lansdorp PM: Collapse of telomere homeostasis in hematopoietic cells caused by heterozygous mutations in telomerase genes. PLoS Genet 8: e1002696, 2012.

43. Schröder CP, Wisman GB, de Jong S, et al: Telomere length in breast cancer patients before and after chemotherapy with or without stem cell transplantation. Br J Cancer 84: 1348-1353, 2001.

44. Mirabello L, Garcia-Closas M, Cawthon R, et al: Leukocyte telomere length in a population-based case-control study of ovarian cancer: a pilot study. Cancer Causes Control 21: 77-82, 2010.

45. Engelhardt M, Ozkaynak MF, Drullinsky P, Sandoval C, Tugal O, Jayabose $\mathrm{S}$ and Moore MA: Telomerase activity and telomere length in pediatric patients with malignancies undergoing chemotherapy. Leukemia 12: 13-24, 1998.

46. Franco S, Ozkaynak MF, Sandoval C, Tugal O, Jayabose S, Engelhardt $\mathrm{M}$ and Moore MAS: Telomere dynamics in childhood leukemia and solid tumors: a follow-up study. Leukemia 17: 401-410, 2003.

47. Diker-Cohen T, Uziel O, Szyper-Kravitz M, Shapira H, Natur A and Lahav M: The effect of chemotherapy on telomere dynamics: clinical results and possible mechanisms. Leuk Lymphoma 54: 2023-2029, 2013. 